\title{
Electrodeposition of Metals in Microgravity Conditions
}

\author{
K. Nishikawa ${ }^{1}$, T. Homma ${ }^{2}$, E. Chassaing ${ }^{3}$, M. Rosso ${ }^{1}$ and Y. Fukunaka ${ }^{2,4}$ \\ ${ }^{1}$ Laboratoire de Physique de la Matière Condensée, UMR CNRS 7643, Ecole \\ Polytechnique, 91128 Palaiseau Cedex, France \\ ${ }^{2}$ Dept. of Applied Chemistry, Waseda University, Japan \\ ${ }^{3}$ IRDEP, UMR CNRS 7174, 6 quai Watier, 78401 Chatou Cedex, France \\ ${ }^{4}$ JAXA, ISS Science Project Office, Tsukuba, Japan \\ E-mail: fukunaka@energy.kyoto-u.ac.jp
}

\begin{abstract}
Metal electrodeposition may introduce various morphological variations depending on the electrolytic conditions including cell configurations. For liquid electrolytes, a precise study of these deposits may be complicated by convective motion due to buoyancy. Zero-gravity $(0-\mathrm{G})$ condition provided by drop shaft or parabolic flight gives a straightforward mean to avoid this effect: we present here $0-G$ electrodeposition experiments, which we compare to ground experiments (1-G). Two electrochemical systems were studied by laser interferometry, allowing to measure the concentration variations in the electrolyte: copper deposition from copper sulfate aqueous solution and lithium deposition from an ionic liquid containing LiTFSI. For copper, concentration variations were in good agreement with theory. For lithium, an apparent induction time was observed for the concentration evolution at 1-G: due to this induction time and to the low diffusion coefficient in ionic liquid, the concentration variations were hardly measurable in the parabolic flight $0-\mathrm{G}$ periods of 20 seconds.
\end{abstract}

\section{Introduction}

Electrochemical interfacial phenomena in microgravity environments have not been paid much attention by electrochemical scientists and engineers. It is simply because of the apparently successful operated energy as seen even in the case of Apollo the 13th. However, the energy storage and power generation, as well as materials processing, may be planned in the international space station (ISS) and in the projects beyond ISS project after the year 2020. The study of electrochemical interfacial phenomena under microgravity conditions will be likely focused in the future.

Electrodeposition or electrochemical dissolution of metal in aqueous solution is a good subject for primary stage of microgravity electrochemical research. Its reaction mechanism is relatively well understood. Moreover, the electrochemical reaction rate can be easily controlled by changing the current density or potential. A transition from 
smooth metal film to dendrite morphology is sometimes found. From the viewpoint of fundamental electrochemistry, dendritic electrodeposits may provide simple model systems to study Diffusion Limited Aggregation (DLA) phenomena. Furthermore, dendrite growth was intensively studied for practical reasons, because of its detrimental role in battery technology[2]. The surface flatness is a key issue to guarantee a longer reversibility of secondary battery during charging/discharging repetition. Thus, the coupling behavior between the morphological variations and ionic mass transfer rate must be indispensably understood.

However, for liquid electrolytes, and whatever the electrochemical system under concern, a precise study of dendrite growth mechanisms may be complicated by convective motion due to buoyancy: even in thin, quasi-two-dimensional horizontal cells with vertical electrodes, electrodeposition is accompanied by a gravity-induced fluid flow at the electrodes. This effect is due to the electrolyte stratification near both electrodes: it has been extensively studied in the recent literature, both theoretically [4-6] and experimentally [7-11]. Convective motion mixes the electrolyte and tends to homogenize the concentration. The effect depends on cell configuration, salt concentration, and current density [4]. This convection driven by buoyancy was shown to increase the instability of system [11]. Obviously, zero-gravity experiments [12] should give a straightforward mean to avoid this effect: apart from gravity, no other parameter is altered. However, these experiments are relatively difficult to work out and of short duration (around 2-25 s in the most affordable facilities: drop tower and parabolic flight).

JAMIC experiments are described at first in this paper. Then, we present a study of electrodeposition in the zero-gravity environment provided by parabolic flights. This project is supported by the Centre National d'Etudes Spatiales (CNES): it plans copper electrodeposition on copper electrode and lithium electrodeposition on $\mathrm{Ni}$ electrodes in the Airbus A300 from Novespace. During these flights, almost zero-gravity conditions are available over 20 to 25 seconds. The project includes the concentration measurements by laser interferometry, and similar but longer experiments performed at $1-\mathrm{G}$ in the cathode over anode configuration. Both series of experimental results are to be compared with numerical calculations.

\section{Experimental}

\subsection{JAMIC Drop Tower}

A quasi two-dimensional electrolytic cell(Figure1) was used in JAMIC. A $1 \mathrm{~mm}$ 
diam. disk cathode was placed at the center of the cell and a flat ring-shaped anode was placed at the outer edge. The thickness of the cathode made of $1 \mathrm{~mm}$ diam. copper wire was adjusted to $100 \mu \mathrm{m}$ by a polishing technique. The circular periphery area of this disk was used as the effective surface of the cathode. Copper foil of $100 \mu \mathrm{m}$ thickness was perforated to manufacture a flat ring-type anode with a $20 \mathrm{~mm}$ inner diam. Both electrodes were sandwiched by two sheets of slide glass. This electrolytic cell was filled with $0.9 \mathrm{M} \mathrm{CuSO}_{4}$ aqueous solution. Electrolysis was carried out at relatively high and constant current densities in order that the interference fringe pattern would appear soon after starting the electrolysis. Thus, the diffusion layer grows in the radial direction.

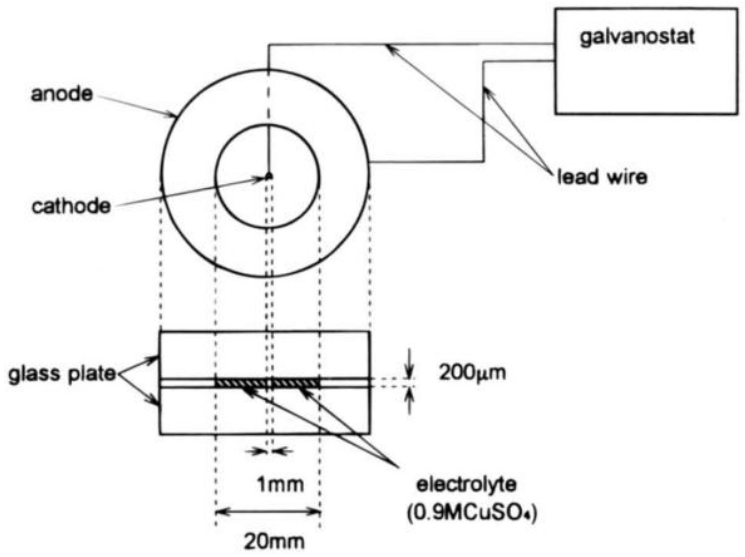

Figure 1. Quasi Two Dimensional Cell with Radial Diffusion Field

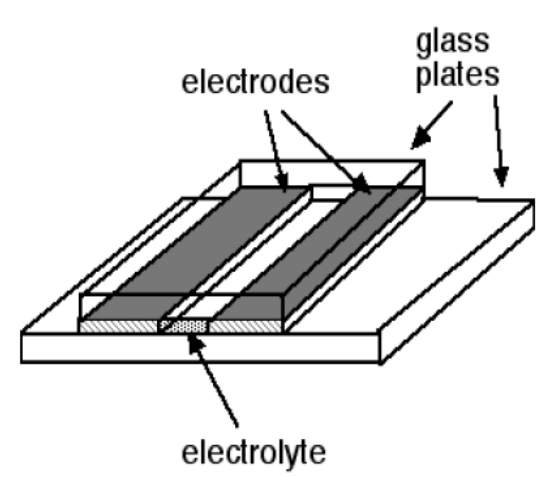

Figure 2 2-D Cell with Linear Diffusion Field

A common path microscopic interferometry was installed in JAMIC drop capsule. Laser diodes of $5 \mathrm{~mW}$ with $680 \mathrm{~nm}$ wavelength and a luminous light-emitting diode were used to observe the interference fringe pattern and the field image, respectively. The electrolytic cell was horizontally installed in this common path interferometry inside a drop capsule. The thickness of electrolyte solution in the cell was measured to be $200 \mu \mathrm{m}$, because both sides of cathode were coated for insulation with polyvinylchloride (PVC). The concentration dependence of refractive index of the electrolyte solution is $0.028 \mathrm{M}^{-1}$ and the resolution power of concentration was, therefore, $5.7 \times 10^{-2} \mathrm{M} \mathrm{CuSO}_{4}$ per fringe.

JAMIC drop capsule started to descend by gradually reducing the electromagnetic current. It was evacuated around the inner capsule, and the outer capsule was accelerated by injecting gas at an appropriate rate to compensate the friction of air inside the drop shaft. A microgravity level less than $10^{-4} \mathrm{G}$ was thus attained $1 \mathrm{~s}$ after disengagement of the capsule. The descending capsule started to decelerate in the braking zone $10 \mathrm{~s}$ after free fall. It experienced about $8 \mathrm{G}$ as a maximum value. The 
electrolysis was, therefore, initiated $1 \mathrm{~s}$ after the capsule had descended the shaft. Electrolysis was then terminated at $9 \mathrm{~s}$ in order to recover the sample electrodeposited only under microgravity conditions.

\subsection{CNES Parabolic Flight}

The project presented here consists in carrying out electrochemical experiments during zero-g periods provided by parabolic flights. The experiments are carried out in a pseudo 2 D-cell (Figure 2). Care is taken to obtain a well defined cell geometry: see Ref. [19] for details. Copper electrodes are used for the copper electrodeposition and nickel electrodes are for lithium electrodeposition: they are held between two glass plates enabling to observe in-situ the electrochemical cell.

The cell has a parallelepipedic shape, with dimensions L, the active length of the electrodes, $l$, the inter-electrode distance, and $\mathrm{d}$, the thickness of the cell, respectively. Practical values are: $\mathrm{L}=0.8-1.5 \mathrm{~cm}, l \approx 0.09-0.3 \mathrm{~cm}, \mathrm{~d} \approx 0.012-0.1 \mathrm{~cm}$. The lateral faces of the electrodes are coated with a hydrophobic film which limits the invasion of the electrolyte between the electrodes and the glass plates. After filling the cell with the electrolyte, it is sealed with a two-component resin before taking off: this allows to keep it under almost constant conditions for several hours [19]. During the flight, the temperature in the plane is kept at $18^{\circ} \mathrm{C}$. Two electrochemical systems are studied: copper deposition from 0.1 and $0.2 \mathrm{~mol} \mathrm{~L}^{-1}$ aqueous $\mathrm{CuSO}_{4}$ solutions and lithium deposition from an ionic liquid.The ionic liquid is $\mathrm{N}$-methoxymethyl-N-methylpyrrolidinium bis (trifluoro-methane-sulfonyl) imide containing $1.0 \mathrm{~mol} \mathrm{~L}^{-1}$ LiTFSI.

In these microgravity experiments, a concentration variation of the electrolyte induced a variation of the refractive index which is measured by a common path laser interferometer. Laser interferometry is a well known technique for measuring the concentration changes in electrochemical processes [20-24]. This study use the interferometer described in Ref. [12] to measure in-situ the variations of ionic concentration in the electrolyte. Because a thin cells $(0.1$ or $1 \mathrm{~mm})$ is used, we expect negligible light deflection due to refractive index gradients [25]. The laser source is a laser diode with $685 \mathrm{~nm}$ wavelength. The change in the refractive index is given by

$$
d \Delta n=S \lambda
$$

where $d$ : the optical path length in the electrochemical cell (here the cell thickness), $\Delta \mathrm{n}$ : 
the change of refractive index, $S$ : the shift of interference fringes, and $\lambda$ : the laser wavelength. The relationship between refractive index and concentration is given by:

$$
\Delta n=\left(\frac{\partial n}{\partial C}\right) \Delta C
$$

We use $(\partial \mathrm{n} / \partial \mathrm{C})=0.0279$ for a $\mathrm{CuSO}_{4}$ aqueous solution and $(\partial \mathrm{n} / \partial \mathrm{C})=0.005$ for LiTFSI in ionic liquid. The transient behaviour of this shift is recorded with a CCD camera. Electrolytic condition is conducted at a constant current or a constant potential.

\section{Results}

\subsection{Copper Electrodeposition in JAMIC Drop Tower}

Figure 3 compares the time variation of interferograms recorded during the electrodeposition of copper at $0.2 \mathrm{~A} \mathrm{~cm}^{-2}$ from $0.9 \mathrm{M} \mathrm{CuSO}_{4}$ solutions obtained under 1- and $0-\mathrm{G}$ conditions. At $1 \mathrm{~s}$ after the start of electrolysis, a single fringe appears around the cathode under 1-G field, whereas no clear image of interference fringe is seen under $0-\mathrm{G}$. At $4 \mathrm{~s}$, the annular fringe pattern under $1-\mathrm{G}$ recedes from the cathode surface, and a fringe appears in the vicinity of the cathode surface under 0-G. After $8 \mathrm{~s}$, two clear interference fringes with broader width are observed in 1-G experiments. At the same time, several fringes with narrower width appear within a distance of $100 \mu \mathrm{m}$ from the cathode surface under $0-G$. It is difficult to count exactly the number of interference fringe with naked eye. The growth of annular interference fringe along the radial direction indicates the development of the diffusion layer of $\mathrm{Cu}^{2+}$ ions, when convection is not induced. The interferogram recorded at $15 \mathrm{~s}$ after the start of electrolysis is also illustrated. The electrolysis has already been terminated, and the capsule has started to decelerate. The less concentrated solution stays near the cathode and slightly concentrated electrolyte does near the anode (with larger surface area). The acceleration force starts to act upon the electrolyte. Then, the lighter electrolyte, constrained inside the diffusional layer adjacent to the cathode, begins to flow to induce a convection loop along two glass sheets. This is a reason why the radius of the annual interference fringe pattern suddenly increased.

In $1-G$ experiments, the fringes are observed at nearly constant intervals along the radial direction. The time variation of $\mathrm{Cu}^{2+}$ ion concentration at the cathode surface is compared with a transient diffusion model. It decreases much slower than the calculated value. The ionic mass-transfer rate from the bulk electrolyte to the cathod surface superimposed by the fluid flow induced even in such a quasi two-dimensional shallow electrolytic cell may partly contribute such a deviation as well as the optical 
deflection error.
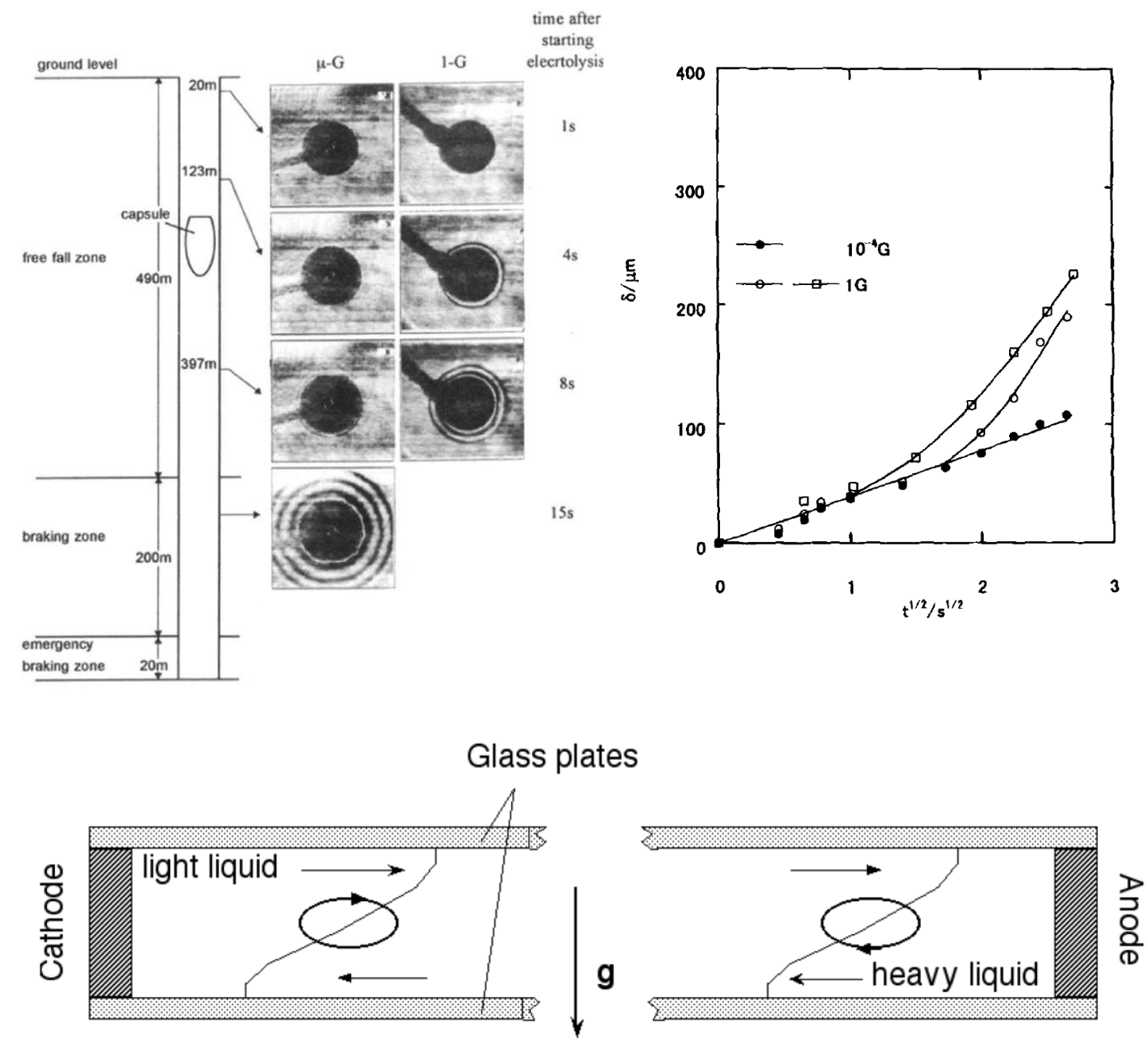

Figure 3 Transient Variations of Interference Fringes and Diffusion Layer Thickness with Progress of $\mathrm{Cu}$ Electrodeposition(0-G and 1-G)

Followed by a Kind of Natural Convection Confined in Quasi-2D Cell in Deccelerated Zone

The laser beam propagates in a straight line in the bulk electrolyte. Considering the resolution power of concentration in the present optical arrangement $(0.057 \mathrm{M}$ per fringe), the outer periphery of the diffusion layer may be conventionally defined as the position where the concentration is decreased by $3 \%$ of the bulk value. Thus, the time variation of the diffusion layer thickness can be reasonably measured. At $0.2 \mathrm{~A} \mathrm{~cm}^{-2}$, the diffusion layer thickness increases proportionally to the square root of time in $0-\mathrm{G}$. The measured diffusion layer thickness in $1-G$ begins to deviate from this linearity at 1 or $3.2 \mathrm{~s}$ after the starting electrolysis, most probably due to induced natural convection. 
The development of diffusion layer thickness under microgravity is analyzed based on the one-dimensional transient diffusion model including the migration effect. Although the physical properties of diffusivity and the transfer number vary with electrolyte composition, constant physical properties of diffusivity and the transfer number are assumed as a first-order approximation. A diffusivity of $5 \times 10^{-6} \mathrm{~cm}^{2} \mathrm{~s}^{-1}$ and a cation transference number of 0.3 are found to give good agreement with measured thicknesses, which are very close to the reported values. ${ }^{22}$

Figure 4 illustrates the potential difference between both electrodes. It almost stays constant at $620 \mathrm{mV}$ for the first $3 \mathrm{~s}$. It suddenly increases by $200 \mathrm{mV}$ around $4 \mathrm{~s}$, followed by a slight increase of potential difference in $0-G$ experiment, while the measured potential difference is slightly raised to $680 \mathrm{mV}$ at $4 \mathrm{~s}$ and remained afterward under 1-G. The abrupt jump in the potential difference may be referred to the increase in concentration overpotential caused by depleted cupric ion at the cathode surface, since the calculated surface concentration of $\mathrm{Cu}^{2+}$ ion reached zero at $3 \mathrm{~s}$. This behavior has been partly observed in the ground level experiment with a horizontal cathode over anode configuration. After the considerable concentration overpotential develops, copper grains grow in a dendritic manner (see Appendix below).

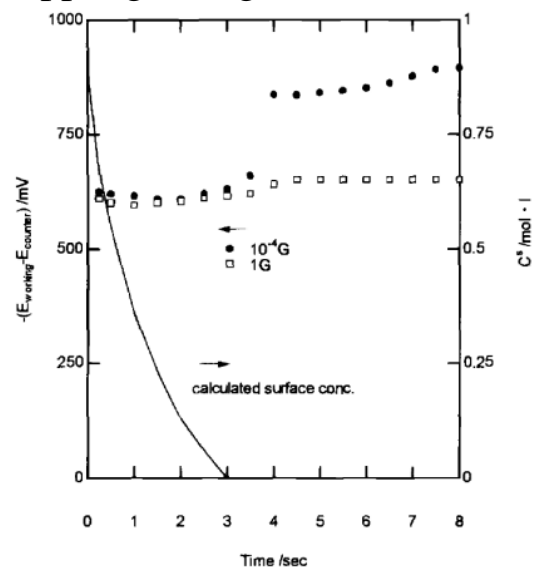

Figure 4 Time Variations of Potential Difference $\left(0.9 \mathrm{M} \mathrm{CuSO}_{4}, 0.3 \mathrm{Acm}^{-2}\right)$

The effect of microgravity on the morphology of copper electrodeposited at 0.05 and $0.3 \mathrm{~A} \mathrm{~cm}^{-2}$ is seen in Figure 5. The electrolysis was conducted over $8 \mathrm{~s}$ under both environments. If copper could be precipitated densely and uniformly on the copper substrate without any voids, the average film thickness would be 0.17 and 1.0 $\mu \mathrm{m}$, respectively. At ground level, a number of $0.5 \mu \mathrm{m}$ diameter grains is uniformly precipitated at both current densities. Since the averaged size of grains is close to the resolution power of the scanning electron microscope (SEM), it is difficult to distinguish any difference on the crystallographic aspect of each grain obtained at both current densities. These most likely grow in a three-dimensional nucleation manner. 
Surprisingly, the significant difference of morphology of copper obtained at the same current density is noticed between $0-\mathrm{G}$ and $1-\mathrm{G}$ level experiments. Some grains grow with preferential growth of the lower index planes at both current densities. A similar difference is also noticed at $0.1 \mathrm{~A} \mathrm{~cm}^{-2}$. At the low current density, the size of larger grains reaches about $2 \mu \mathrm{m}$, which is about four times larger than that for the sample obtained at ground level. The preferential growth of lower index planes is more evident at the lower current density.

It is not clear why such a morphological difference is induced by the different level of gravitational acceleration. As shown above, the surface concentration of $\mathrm{Cu}^{2+}$ ion is more quickly lowered with time under microgravity, so long as the electrolysis conducted at the same current density. The main part of the cathode surface is thus exposed to the electrolyte with lower concentration of $\mathrm{Cu}^{2+}$ ion under microgravity. If we assume that the three dimensional nucleation rate becomes low with decreasing in the surface concentration of $\mathrm{Cu}^{2+}$ ion, we obtain fewer nuclei on the cathode under microgravity. When the electrolysis is conducted to produce the same amount of charge, more coulombic charge should be distributed on the particular grain to introduce the larger-sized grains.

$0.3 \mathrm{~A} / \mathrm{om}^{2}$
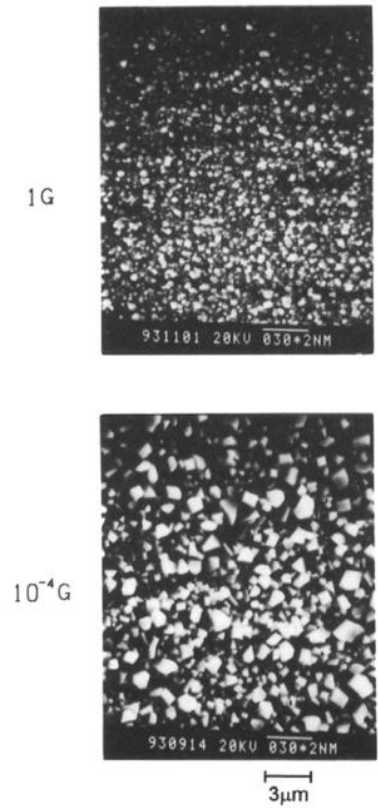

$0.05 \mathrm{~A} / \mathrm{cm}^{2}$
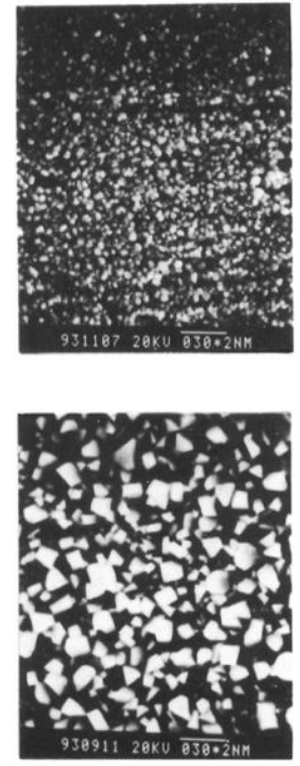

Figure 5. Comparison of Morphology of Copper Electrodeposited in Terrestrial and Microgravity Experiment: Scratched Traces by Sandpaper are Visible on $\mathrm{Cu}$ Disk Substrate Surface with $100 \mu \mathrm{m}$ Thickness. $\left(0.9 \mathrm{M} \mathrm{CuSO}_{4}\right.$ Aqueous Solution, Electrodeposition Duration Period of $8 \mathrm{~s}$, 0.05 and $0.3 \mathrm{~A} \mathrm{~cm}^{-2}$ )

However, the electrical conductivity is simultaneously reduced with lowered concentration of $\mathrm{Cu}^{2+}$ ions near the cathode. It may considerably influence the ionic mass transfer rate due to the migration effect. ${ }^{25}$ Thus, the concentration gradient of $\mathrm{Cu}^{2+}$ ion near the cathode is no longer the same as that observed at the initial stage of 
electrolysis. Further measurement of $\mathrm{Cu}^{2+}$ ion concentration profile is necessary to understand the morphological variations introduced by the different level of the gravitational acceleration force.

\subsection{Electrodeposition of Metals in Parabolic Flight}

\subsubsection{Copper Electrodeposition}

The concentration profile in the electrolyte for various current density and bulk concentrations were measured. Figure 6 shows an example of interference fringes recorded before and 15 seconds after the beginning of cell polarization. The fringes are bent in the vicinity of the cathode, at the bottom of the Figure, because of the variation of concentration. These concentration variations were also numerically calculated, using general equations describing transport phenomena in electrochemical systems [30], and taking into account the variation of the diffusion coefficients with concentration in $\mathrm{CuSO}_{4}$ aqueous solution [31]. The time variation of surface concentration during and after one parabola is shown in Figure 7, and compared with theory. A good agreement between experimental and theoretical results was found. After the end of the parabola, the concentration at the cathode rapidly returned to its initial value: this was due to the large $\mathrm{G}(\sim 1.5 \mathrm{G})$ imposed at this time in order to drive back the plane to its normal flying conditions.

We have reported elsewhere [28] the concentration measurements obtained in 1-G conditions in a C/A cell. A similar agreement with theory was found for measurements performed before the onset of dendritic growth. On the other hand, in experiments performed in horizontal cells [4, 7-10], the concentration variations were markedly different.

We did not observe dendritic growth of copper, however, because the dendrites only appear after an induction time $t_{\mathrm{s}}$ the so-called Sand time [29]. Sand time varies as

$$
t_{S}=\frac{\pi e^{2} D\left(1+\mu_{C} / \mu_{a}\right) C_{0}^{2}}{J^{2}}
$$

where $\mathrm{e}$ is the electronic charge, $\mathrm{D}$ the diffusion constant, $\mu_{\mathrm{c}}$ and $\mu_{\mathrm{a}}$ the cationic and anionic mobilities respectively, $\mathrm{C}_{0}$ is the initial ionic concentration and $\mathrm{J}$ the current density. To obtain a Sand time shorter than 10 seconds for example, would require a high current density (larger than $20 \mathrm{~mA} \mathrm{~cm}^{-2}$ ) and still, the time available for observing dendritic growth would be very short. Experimental environment did not provide such conditions in this parabolic flight campaign. Further experiments are thus necessary to explore dendritic growth itself. 

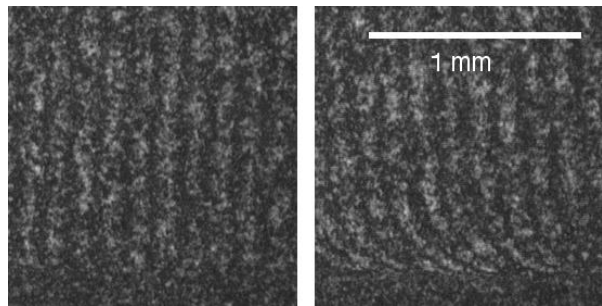

Figure 6. Interference Fringes before of and $15 \mathrm{~s}$ after $\mathrm{Cu}$ Electrodeposition. (0.1 $\mathrm{mol} \mathrm{L}^{-1} ; 8 \mathrm{~mA} \mathrm{~cm}^{-2}$;

Cell Thickness is $0.1 \mathrm{~cm}$.)

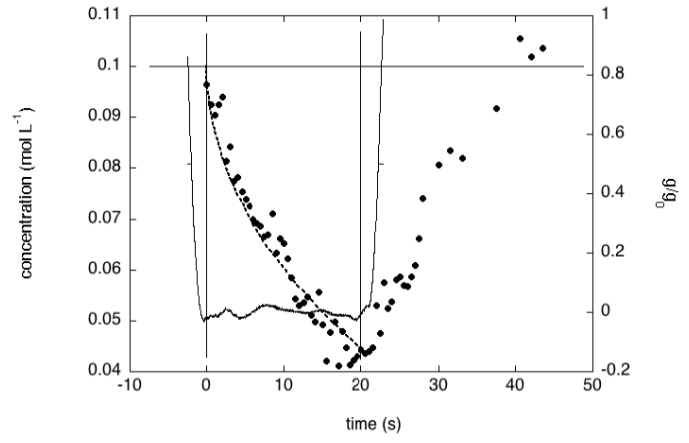

Figure 7. Surface Concentration Variations $\mathrm{Cu}^{2+}$ Ion during Parabolic Flight (Closed Circles: Measured, Dashed Line: Calculated)

\subsubsection{Lithium Electrodeposition}

As mentioned earlier, a series of experiments were performed at 1-G. A typical result is given in Figure 8, showing the variation of the cathode surface concentration as a function of the square root of time. In this experiment, the applied current density is $1 \mathrm{~mA} \mathrm{~cm}{ }^{-2}$ and cell thickness is $\mathrm{d}=0.1 \mathrm{~cm}$. One clearly sees a first stage, where the concentration variation is very small. Then, after $90 \mathrm{~s}$, the variation is much more rapid. Such an induction time was already reported [16]. In the present case, the observed induction time might be due to side reactions of the electrodeposited lithium metal. The diffusion coefficients in the ionic liquids are very low: from the variation of the diffusion layer thickness in the experiment illustrated in Figure 8, we estimated a diffusion coefficient $\mathrm{D} \sim 10^{-7} \mathrm{~cm}^{2} \mathrm{~s}^{-1}$. This value is in good agreement with that reported by other methods [32]. The concentration variations are compared in Figure 8 with theoretical values [30], which are calculated without taking into account the concentration dependence of the diffusion coefficient. The result obtained for the above value of $\mathrm{D}$ and a transport number of 0.35 for $\mathrm{Li}^{+}$ion is shown in Figure 8 as a straight continuous line.

Due to the very low diffusion coefficient and to the induction time mentioned above, the concentration variations due to $\mathrm{Li}$ deposition during the first 20 seconds are very small, and the concentration boundary layer thickness is at most $10 \mu \mathrm{m}$ : thus concentration variations are hardly detectable by our interferometer. The Li deposition experiment would thus require longer zero-g times. 


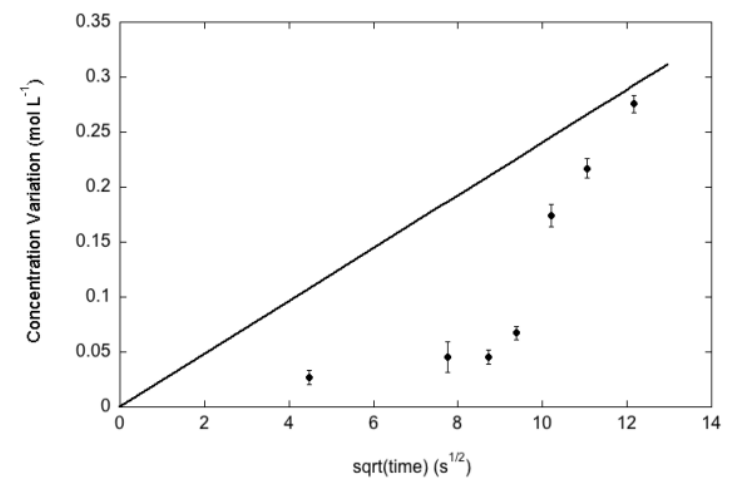

Figure 8. Li Electrodeposition in Ionic Liquid: Time Variations of Cathode Surface Concentration with Electrolysis(Experimental: Circles, Calculated: Straight Line).

\section{Conclusion}

A common path microscopic interferometry was used in a drop shaft experiment. The growth of the diffusion boundary layer of $\mathrm{Cu}^{2+}$ ions associated with electrodeposition of copper in $\mathrm{CuSO}_{4}$ solution was successfully measured in situ under microgravity. When the electrolysis is conducted at $1-\mathrm{G}$, natural convection is induced even in such a shallow electrolyte layer of $200 \mu \mathrm{m}$ thick. Meaurements of voltage drop betwen both electrodes show the abrupt increase in potential difference, just after the surface concentration of $\mathrm{Cu}^{2+}$ ion reaches zero under 0-G. A significant difference of morphology appears after only 8s of electrodeposition at constant current density. Larger grains with preferential growth of lower indexes are obtained in 0-G. The coupling effect of the diffusion and migration mechanism to the electrocrystallization phenomena under $0-\mathrm{G}$ must be further examined. It is necessary to monitor the transient variation of cathodic overpotential with the progress of electrodeposition.

Although of limited duration, parabolic flight experiments can provide interesting results on the early stage of electrodeposition. The evolution of concentration profiles is consistent with the theoretical calculation curves. However, a more complete study would require further zero-g experiments: for studying dendritic growth of copper, or for deposition of lithium from ionic liquids, longer times would be necessary (such as those available in sound rockets or in the International Space Station). These improvements are considered in the framework of a project developed by an "Electrochemical Nucleation \& Growth" ESA Topical Team.

\section{References}

[1] Witten T A and Sander L M 1981 Phys. Rev. Lett. 471400

[2] Tarascon J-M and Armand M 2008 Nature 451652 
[3] Laitinen H A and Kolthoff I M 1939 J. Am. Chem. Soc. 613344

[4] Chazalviel J-N, Rosso M, Chassaing E and Fleury V 1996 J. Electroanal. Chem. 40761

[5] Marshall G, Mocskos P, Swinney H L and Huth J M 1999 Phys. Rev. E 592157

[6] Kawai S, Nishikawa K, Fukunaka Y and Kida S 2007 Electrochim. Acta 53257

[7] Rosso M, Chazalviel J-N, Fleury V and Chassaing E 1994 Electrochim. Acta 39507

[8] Barkey D P, Watt D, Liu Z and Raber S 1994 J. Electrochem. Soc. 1411206

[9] Huth J M, Swinney H L, Mc Cornmick W D, Kuhn A and Argoul F 1995 Phys. Rev. E 513444

[10] Argoul F, Freysz E, Kuhn A, Léger C and Potin L 1996 Phys. Rev. E 531777

[11] Marshall G, Mocskos E, González G, Dengra S, Molina F V and Iemmi C 2006 Electrochim.Acta $\mathbf{5 1} 3058$

[12] Fukunaka Y, Okano K, Tomii Y, Asaki Z and Kuribayashi K 1998 J. Electrochem. Soc. 1451876

[13] González G, Marshall G, Molina F and Dengra S 2002 Phys. Rev. E 65051607

[14] Marshall G, Perone E, Tarela P and Mocskos P 1995 Solitons and Fractals 6315

[15] Sagués F, López-Salvans M Q and Claret J 2000 Phys. Reports 33797

[16] Nishikawa K, Fukunaka Y, Sakka T, Ogata Y H and Selman J R 2007 Electrochim. Acta, $\mathbf{5 3} 218$

[17] González G, Rosso M, Chassaing E and Chazalviel J-N 2007 Electrochim. Acta 53 141

[18] Morisue M, Fukunaka Y, Kusaka E, Ishii R and Kuribayashi K 2003 J. Electroanal. Chem. 559155

[19] Rosso M, Chassaing E, Chazalviel J-N and Gobron T 2002 Electrochim. Acta 47 1267

[20] McLarnon F R, Muller R H and Tobias C W 1976 Electrochim. Acta 21101

[21] Denpo K, Okumura T, Fukunaka Y and Kondo Y 1985 J. Electrochem. Soc 132, 1145

[22] Eriksson R 1995 Electrochim. Acta 40725

[23] Konishi Y, Tanaka Y, Kondo Y and Fukunaka Y 2000 Electrochim. Acta 46681

[24] Fukunaka Y, Nakamura Y and Konishi Y 1998 J. Electrochem. Soc. 1453814

[25] Beach KW, Müller R H and Tobias C W 1973 J. Opt. Soc. Am. A 63559

[26] Hawkes J B, Astheimer R W 1948 J. Opt. Soc. Am. 38804

[27] Nishikawa K, private communication

[28] Nishikawa K, Chassaing E and Rosso M 2011 Electrochim. Acta 565464

[29] Sand H J S 1901 Phil Mag 145 
[30] Marciano S L and Arvia A J 1983 Diffusion in the absence of convection: steady state and nonsteady state Comprehensive Treatise of Electrochemistry Ed Yeager E, Bockris J O'M, Conway B E, Sarangapani S (New York: Plenum Press) vol.6, pp. 65-132,

[31] Miller D G, Rard J A, Eppstein L B and Robinson R A 1980 J. Solution Chem. 9 467

[32] Nishikawa K, Naito H, Kawase M and Nishida T 2011 Lithium Batteries Discussion Arcachon (France)

\section{Acknowledgements}

The authors thank Drs. H. Yasuda (Osaka University), Y. Inatomi (Japan Aerospace Exploration Agency - JAXA), K. Kinoshita (JAXA), M. Takayanagi (JAXA), S. Yoda (JAXA) and T. Maki (Olympus Corporation), for providing the interferometer especially designed for zero-g experiments that we used in this study. The authors also wish to thank Dominique Clément for the mechanical realization of the experimental setup and for the many technical solutions which he brought to the problems involved in the operation of this experiment in parabolic flight. Centre National d'Etudes Spatiales is acknowledged for its financial support and Novespace for help in preparing the experiments. 